\title{
INTUBATION STUDIES OF THE HUMAN SMALL INTESTINE. XXIII. A METHOD OF DETERMINING DIGESTIVE ACTIVITY IN ANY PORTION OF THE GASTRO-INTESTINAL TRACT, WITH SOME MEASUREMENTS OF PROTEIN DIGES- TION IN THE STOMACH AND SMALL INTESTINE ${ }^{1,2}$
}

\author{
By KENDALL A. ELSOM, FRANCIS W. CHORNOCK, and FRANCIS G. DICKEY \\ (From the Gastro-Intestinal Section (Kinsey-Thomas Foundation) of the Medical Clinic, \\ Hospital of the University of Pennsylvania, Philadelphia)
}

(Received for publication May 21, 1942)

Two essential physiological processes occur in the small intestine-the enzymatic cleavage of complex food substances into simpler nutritive compounds, and their transport through the intestinal wall into the body. Abnormality in one or both of these processes accounts in large part for the nutritional disturbance observed in many disorders, such as sprue, pancreatic disease, certain avitaminoses, regional enteritis, and ulcerative colitis. It is obviously desirable to have clinical methods of measuring each of these functions separately since a given disease may be due either to disturbance of digestion or of absorption, or both. The available tests of digestive function consist of "in vitro" determinations of the enzymatic activity of gastric or duodenal juice, and of the examination of the feces for undigested food material. Neither method is wholly satisfactory. The latter is relatively crude, it gives no quantitative data and the result is greatly influenced, not only by enzymatic activity, but also by the speed of passage of the intestinal contents. The former has the limitation of indicating only whether enzymes are present in the upper intestinal tract but does not disclose whether or not the conditions are favorable for their activity in the distal portions of the bowel.

It seemed to us that an improved method for studying digestion might be developed now that small intestinal intubation has become a standard procedure. We have therefore devised an apparatus, to be described presently, which utilizes this method and which makes it possible to test di-

\footnotetext{
1 Presented in abstract before the American Society for Clinical Investigation. Atlantic City, N. J., May 4, 1942.

2 Aided by a grant from the Smith, Kline and French Laboratories, Philadelphia.
}

rectly the digestion of suitable solid food materials, in any portion of the human gastrointestinal tract. The apparatus is so constructed that the test food stuff is exposed to the digestive enzymes only over a designated period and in that area of the bowel selected for study. The method has in our opinion certain points of superiority over those previously employed. Measurements can be made anywhere in the intestinal tract, the test object remains in a constantly renewed bath of intestinal juice while the products of enzyme reactions are carried away, thus avoiding their known retarding effect on farther enzyme activity. The method obviates the withdrawal of fluid for analysis and hence avoids the possible inactivation of enzymes which follows exposure to light, oxidation, and alterations in temperature and in $\mathrm{pH}$. The present communication describes the apparatus and technique of its employment, and gives data on the digestion of protein in the normal and achlorhydric stomach and at various levels of the normal small intestine.

\section{METHOD}

The apparatus ${ }^{8}$ (Figure 1) consists of a cylinder of thin brass tubing $(a), 4 \mathrm{~cm}$. long and $1 \mathrm{~cm}$. in outside diameter, the proximal end of which is narrowed and flanged for the attachment of a rubber tube $(e)$. This cylinder serves as a housing for an inner brass cylinder (b) of equal length, which fits closely but may move freely within it. The inner cylinder is occluded at its proximal extremity by a piston-like disc, its walls are fenestrated in the distal $3 \mathrm{~cm}$., and its exposed extremity is equipped with a screw cap (c) which permits the introduction of a test food substance weighing approximately 1 gram. By exerting air pressure through the rubber tube $(e)$, the inner cylinder, wholly sheathed in

\footnotetext{
8 We are indebted to Mr. Carol Kelly for constructing the apparatus, as well as for valuable suggestions concerning its design.
} 

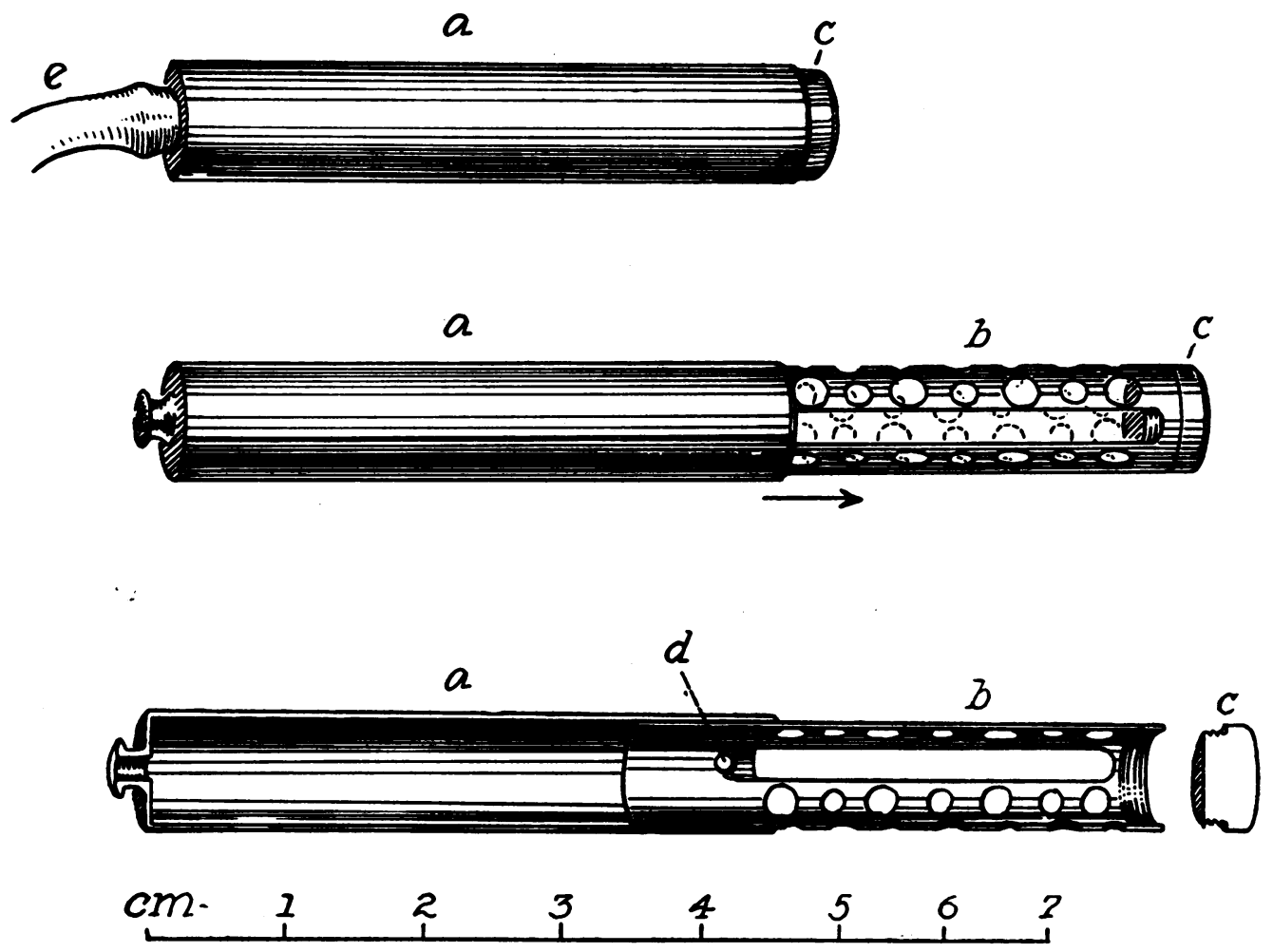

Fig. 1. The Apparatus for Measuring the Digestion of a Test Food Substance

the housing, may be ejected so that its fenestrated distal portion protrudes from the open end of the housing, which contains in its wall a check pin $(d)$ to prevent total expulsion of the inner cylinder. Sufficient friction is present between the two cylinders then to maintain the inner cylinder in its ejected position.

\section{TECHNIQUE}

Observations were begun in the morning, after an overnight fast. Depending on the portion of the intestinal tract to be studied, the apparatus described was attached either to a single lumen tube customarily used for duodenal intubation, or to one lumen of a MillerAbbott tube, the other lumen of which was connected to an inflatable balloon. Into the inner cylinder of the apparatus was placed the carefully weighed test object and the cylinder was then retracted into the housing. The distal tip of the apparatus was dipped into melted paraffin which, when it cooled, sealed off the test object from contact with gastric or intestinal juice. With fluoroscopic guidance, the tube was then introduced to any portion of the intestinal tract, by the technique of intubation repeatedly described from this clinic. When the apparatus was in the desired position, the balloon was deflated and the inner cylinder ejected by means of air pressure. The apparatus then remained stationary, or advanced at most a few inches, permitting contact of the contained test material with the digestive enzymes. At the end of a measured period of time, the tube and attached apparatus were withdrawn, either with the fenestrated cylinder in its ejected position or, if possible, retracted into the housing.

\section{HISTORICAL}

The ingestion of a food substance, usually in a fenestrated container, with subsequent observations of its condition after it had traversed the intestinal tract, is an experimental principle employed in the earliest demonstrations of digestive activity. Spallanzani (1), in studying digestion in a variety of animals, “. . . employed hollow globules of brass half an inch in diameter and pierced like a sieve, which I could open and shut at pleasure by means of a screw worked upon the edge of the two hemispheres into which each globule was divisible."

In an inaugural dissertation (Edinburgh, 1777) which is published as an appendix to Spallanżani's Dissertations, Stevens (2) describes observations in which he gave the subject of his experiments "a hollow silver sphere, divided into two cavities by a partition and perforated on the surface with a great number of holes, capable of admitting a needle: into one of these cavities was put four scruples and a half of raw beef, and into the other five scruples of raw bleak. The sphere was voided in twenty-one hours, when the beef was found to have lost one scruple and a half and the fish two scruples.... I procured another sphere with holes so large as to receive a crow's quill, and enclosed some beef a little masticated 
into it. It was voided quite empty thirty-eight hours after it was swallowed." 4

\section{OBSERVATIONS ON PROTEIN DIGESTION}

In the observations to be reported here, the described method was adapted for a study of protein digestion. Pork heart muscle was selected as a test object since it is easily obtainable in fresh condition after government inspection, is remarkably constant in protein content, ${ }^{5}$ is practically free of fat and fibrous tissue, and will not physically disintegrate and thus be lost from the fenestrated cylinder. For our purposes, raw muscle proved superior to dried or cooked muscle. The technique described above was employed in every detail. The test object was a columnar piece of heart muscle, weight approximately 0.8 gram.

The amount of digestion occurring after a 3hour test was determined as follows: A portion of the heart muscle, immediately adjacent to the sample used in the capsule, was weighed and analyzed for nitrogen by a micro-Kjeldahl method. This permitted a calculation of the total nitrogen content of the sample employed in the test. At the conclusion of the observation, the nitrogen content of the tissue remaining in the capsule was determined, and the difference represented the amount of protein which had been digested. This is expressed as the percentage of that present in the original test object.

The gastric digestion of pork heart muscle was tested 13 times in 10 subjects without gastrointestinal disease and with a normal concentration of gastric acid. Ten subjects from the hospital wards or out-patient clinics, known to have true achlorhydria, were similarly tested. In all cases, the position of the tube in the stomach was determined fluoroscopically. Two professional subjects in good health were employed for the observations on the small intestine, of which 33 were made-11 in the duodenum, 13 in the jejunum, and 9 in the ileum.

4 We can confirm this observation of Stevens'. On one occasion the apparatus became detached from the tube while in the stomach and was permitted to proceed through the intestinal tract. When recovered approximately thirty-six hours later it was empty.

B In 67 observations, the average protein content of pork heart muscle was 17 per cent, the maximum figure 19 per cent, and the minimum 16 per cent.
In order to determine the relationship between the time of exposure of the test object and the amount of digestion which occurred, measurements were made in the duodenum of a normal subject for periods of 30 to 240 minutes.

In order to discover whether the proteolytic action observed in a 3-hour test is due to the enzymes already present in the bowel, or depends upon the secretion of an additional supply, we have carried out, in a normal subject, experiments in which the pancreatic juice formed after the beginning of the observation was prevented from reaching the test object. This was accomplished (Figure 2) by the use of an inflated balloon (a) which occluded the lumen of the bowel, immediately proximal to which were perforations $(b)$ through which constant suction was exerted by a Wangensteen apparatus, and 12 inches distal to the balloon was the digestion apparatus $(c)$. The tube was introduced so that the balloon was in the distal duodenum or proximal jejunum. After obstructing the bowel by distending the balloon, continuous suction was applied withdrawing all contents from the bowel lumen proximal to it. The test substance was then exposed. This double procedure of occlusion and suction effectively

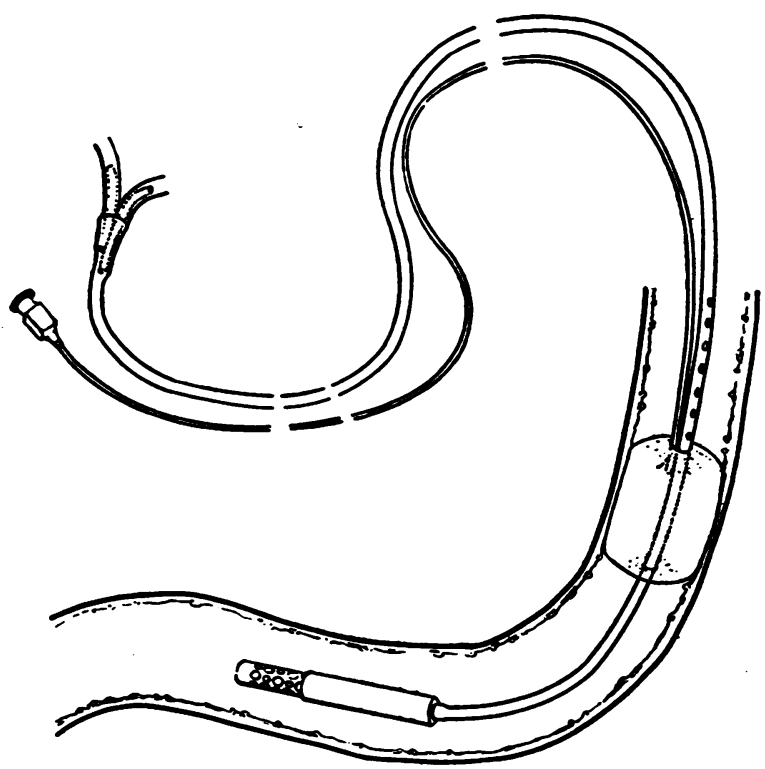

Fig. 2. The Arrangement of the Apparatus for Obstructing the Bowel Proximal to the Test ObJECT

Constant suction was applied through the perforations proximal to the occluding balloon. 
prevents leakage of material past the balloon, as we have repeatedly demonstrated, using either a thin suspension of barium or a dilute solution of vital red.

In 3 observations, we attempted, by preliminary lavage, to remove the enzymes already present in the bowel before exposing the test substance. For this purpose, we used water, $\mathrm{N} / 20 \mathrm{HCl}$, and dilute suspension of colloidal aluminum hydroxide.

\section{RESULTS}

\section{Stomach}

The measurements on the gastric digestion of protein in the normal and achlorhydric subjects are given in Figure 3. In the normal subjects, the average figure was 53 per cent, which ex-

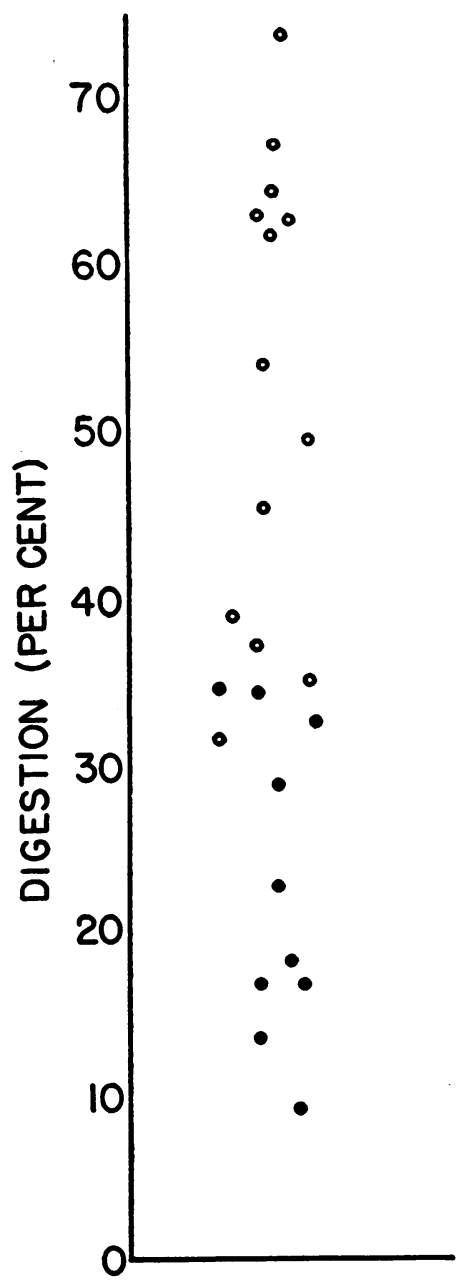

Fig. 3. The Gastric Digestion of Protein in NorMAL (OPEN CIRCLES) AND ACHLORHYDRIC (CLOSED CIRCles) Subjects

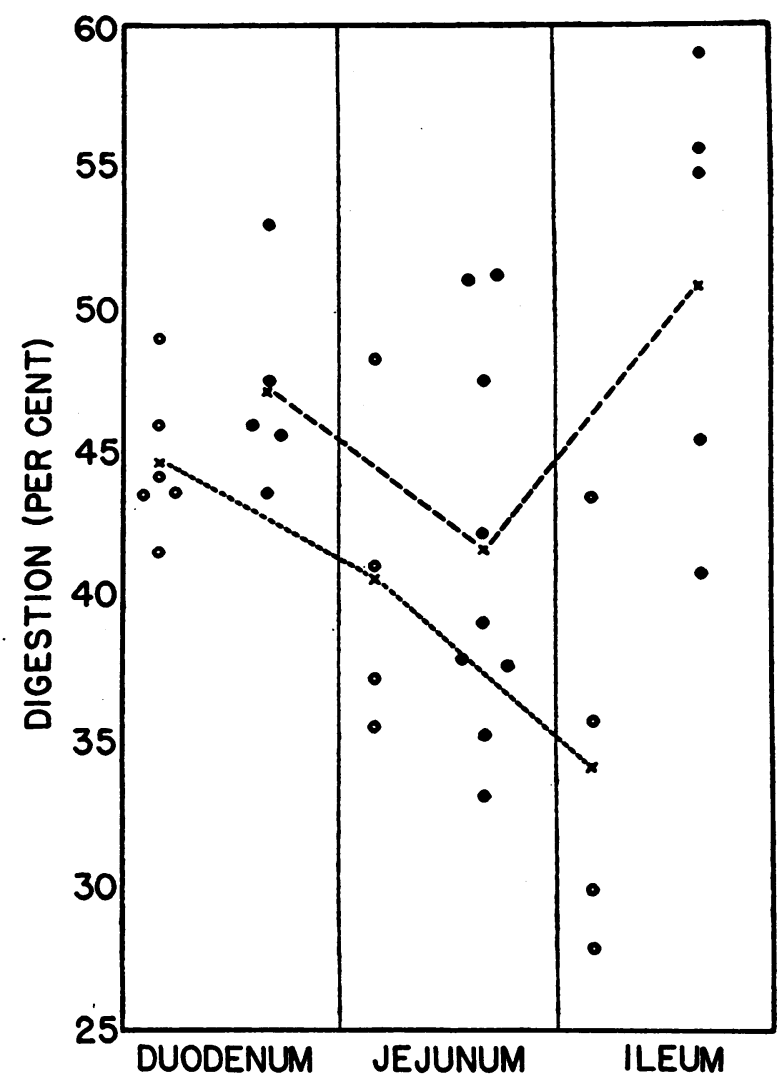

Fig. 4. The Digestion of Protein at Different Levels of the Small Intestine in 2 Normal SubJECTS

Subject 1 (open circles), Subject 2 (closed circles). The broken lines join the average figures.

ceeds that for any other portion of the gastrointestinal tract. The individual variation was great, and in a single subject, tested 4 times, the results varied between 45 and 74 per cent. In the achlorhydric group, 1 subject had Addisonian anemia, with 16 per cent digestion. The others had no recognizable organic lesions of the stomach. The average figure was 23 per cent, the extremes were 9 and 35 per cent.

The results of the 33 observations made on 2 normal subjects, 1 female (Subject 1) and 1 male (Subject 2), are shown in Figure 4. Each point represents a single observation, made in that portion of the small intestine indicated in the figure. The points joined by lines show the averages for both subjects. It will be observed that the most constant results were obtained in the duodenum (average 45 per cent), while greater variation occurred in the jejunum (average 41 per cent) 


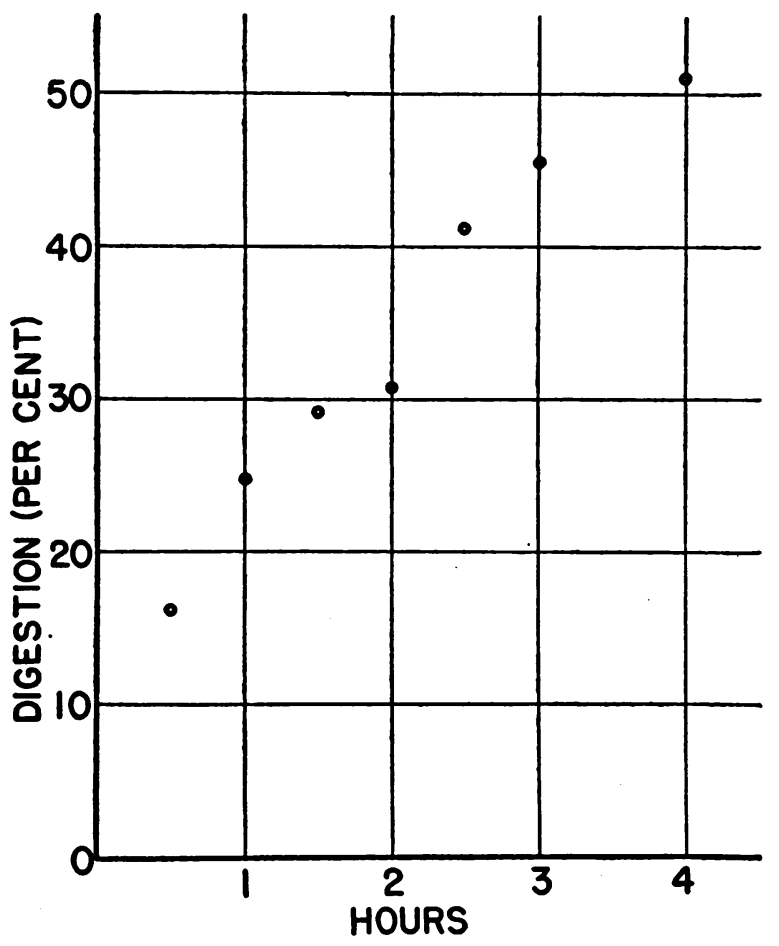

Fig. 5. The Relationship Between the Time of Exposure and the Digestion of Protein in the Duodenum of a Normal Subject

and ileum (average 42 per cent). The average readings for the 2 subjects were almost identical, both in the duodenum and in the jejunum. In the ileum, however, the results in the 2 subjects differed widely; Subject 1 digested, on the average, 31 per cent of the test protein, while Subject 2 digested 50 per cent.

The results of testing the digestion of heart muscle at half-hourly intervals up to 4 hours are shown in Figure 5. The readings so obtained arrange themselves along a parabolic curve, and when their logarithms are plotted, a straight line results. This type of curve characterizes the general reaction of a single enzyme and substrate.

\section{The exclusion of digestive juice from the test object}

In one observation, digestion was measured in the upper jejunum. No effort was made to remove the enzymes already present, but all intestinal content proximal to the area studied was withdrawn by constant suction during the period of observation. Forty-four per cent of the test object was digested in 3 hours. This is essentially the figure which had been obtained on previous tests in this exact location, when the normal current of intestinal juice had not been diverted. In 3 observations, efforts were made, by careful preliminary lavage, to remove or inactivate all enzymes present in the segment of bowel to be studied. Water, $\mathrm{N} / 20 \mathrm{HCl}$, and dilute suspension of an adsorbant, colloidal aluminum hydroxide, were each used once, for periods of lavage from one-half to one hour, in amounts from 250 to $800 \mathrm{cc}$. This procedure reduced the digestion only moderately, to 29,28 , and 36 per cent respectively. This is in agreement with the observations of Owles (3), who found that prolonged washing of the jejunum was required to remove previously secreted erepsin.

\section{DISCUSSION}

It may be objected that, in this method of measuring digestive activity, the presence of the tube and metal apparatus produces mechanical irritation, which is known (3) to influence the secretion of enzymes. This possibility cannot be ignored, but it appears to be a difficulty inherent in any method now known. The method gives no information concerning the extent to which the substance being studied is broken down by enzymatic activity. In the observations on protein digestion, described here, the ultimate degree of proteolysis is undetermined. When the enzyme activity at the surface of the test object has produced a soluble protein component, that material is lost from the test object, regardless of the degree of digestion which has occurred. For example, the test object might be equally reduced in weight by the formation, in one case, of soluble acid metaprotein, formed by the action of gastric $\mathrm{HCl}$, and in another, by the solution of products of tryptic activity in the duodenum. The test would indicate an equal amount of digestion, although, in the latter case, the cleavage of the protein molecule would be much greater. However, if its inherent limitations are taken into proper account in the interpretation of the results, the method appears to provide a useful and simple procedure for measuring digestion in portions of the human intestinal tract which, until recently, have been sufficiently inaccessible to escape careful study.

The general method has thus far been adapted only to a study of protein digestion. Suitable test 
objects to measure the carbohydrate and fat splitting power of the digestive juice can undoubtedly be developed, and, it is hoped, a satisfactory test substance containing all three major foodstuffs may be found, thus obviating the limitation of testing only one substance at a time.

The results of the study of protein digestion in the stomach are in accord with many earlier observations, the most familiar of which are those of Beaumont (4). They indicate that a very considerable proteolytic cleavage can occur in this organ. The conditions of this test, in which meat alone is introduced, are, of course, somewhat unusual. The recent observations of Beazell (5) show that when a protein and carbohydrate meal is ingested, gastric digestion of carbohydrate is considerable, while that of protein is negligible. However, the conditions are so different in the two types of experiments that the results are not necessarily conflicting. The relative incapacity of the achlorhydric stomach to digest protein is probably due to deficiency both in acid and in enzyme secretion, for it is known (6) that in this condition the peptic, as well as acid, secretion is often deficient, and even though it were not, the $\mathrm{pH}$ of the achlorhydric stomach is not optimal for peptic activity.

The digestion of protein which, as we have shown, can begin in the ileum is presumably due to the presence of pancreatic trypsin which has been carried in active form into the distal bowel: This must be true, since it is known that the only other proteolytic enzyme there, the erepsin of the succus entericus, does not attack native proteins, but acts only on polypeptides and dipeptides. The further fact appears that a sufficient quantity of protein-splitting enzymes is present throughout the gut in the fasting state, so that even though additional amounts are prevented from reaching the distal bowel during the period of study, a very considerable breakdown of protein occurs. The observation, therefore, that the digestion of protein can begin in the distal segments of the bowel, without any preliminary proteolysis in the upper intestine, and the demonstration that this digestion can be carried out solely by the enzymes already present, even though the subject has fasted for 18 hours, emphasizes the fact that the normal digestive tract possesses a very impressive margin of safety with respect to the breakdown of protein foods. These observations aid in explaining the sometimes remarkably complete digestion which occurs in clinical cases where large segments of the small bowel have been resected, where marked small intestinal hypermotility exists, or where entero-enteric fistulae have shortened the course taken by the intestinal contents.

\section{SUMMARY}

A method has been developed for measuring digestive activity at any level of the human gastrointestinal tract. A specially devised apparatus containing a test food substance is introduced into the intestine by intubation. When it has reached the desired position, the test substance is exposed to the action of the intestinal juice. The digestion which occurs in a measured time can be determined by appropriate analysis of the remaining portion of the test substance. We have adapted the method to a study of protein digestion, using pork heart muscle as the test material. The results indicate that substantial proteolytic activity occurs in the normal stomach, far less in the achlorhydric stomach. Throughout the normal small intestine, the concentration of proteolytic enzymes is sufficiently high to effect a very considerable amount of digestion, regardless of the point at which the process begins, or whether a further supply of enzymes is available during the period of the test. The method should prove useful in studying clinical problems which involve abnormalities in digestion.

We are indebted to Dr. Walter G. Karr for many helpful suggestions, and to Miss Thelma Cline, R.N., for assistance in the intubation.

\section{BIBLIOGRAPHY}

1. Spallanzani, Abbé Lazarus, Dissertations Relative to the Natural History of Animals and Vegetables. London, 1799, Vol. I.

2. Ibid. Appendix, p. 375.

3. Owles, W. H.: Investigations of the functions of the small intestine in man by intestinal intubation. Clin. Sc., 1937, 3, 21.

4. Beaumont, William, Experiments and Observations on the Gastric Juice. Plattsburgh, 1833.

5. Beazell, J. M., A re-examination of the role of the stomach in the digestion of carbohydrate and protein. Am. J. Physiol., 1941, 132, 42.

6. Rasmussen, R. A., and Brunschwig, A., Peptic activity of achlorhydric human gastric juices from carcinomatous stomachs. A comparative study. Proc. Soc. Exper. Biol. and Med., 1941, 46, 298. 